\title{
COGNITIVE IMPAIRMENT IN PATIENTS WITH BIPOLAR DISORDER IN REMISSION
}

\author{
Y. Ashenbrenner $^{1,2}$, E. Chumakov ${ }^{1,3}$, N. Petrova ${ }^{1}$.
}

${ }^{1}$ Saint-Petersburg State University, Department of Psychiatry and Addictions, Saint-Petersburg, Russia. ${ }^{2}$ St.-Petersburg Psychiatric Hospital No 1 named after P.P. Kaschenko, Day hospital No 3, Saint-Petersburg, Russia. ${ }^{3}$ St.-Petersburg Psychiatric Hospital No 1 named after P.P. Kaschenko, Day hospital No 1, Saint-Petersburg, Russia.

Background: Many patients with bipolar disorder (BD) experience cognitive impairment $(\mathrm{CI})$ in remission. The relationship of $\mathrm{CI}$ in patients with $\mathrm{BD}$ in remission with the presence of residual symptoms is not completely clear.

Aims. To assess the level of $\mathrm{CI}$ in patients with BD in remission.

Methods. The sample consisted of 64 adults with BD in remission (according to the DSM-V criteria), the average age $-36.77 \pm 5.81$. The onset of the disorder $-27.44 \pm 4.06$ years, the average duration of the disorder $-9.33 \pm 4.39$ years. We provided a single examination of a patient including clinical interview and screening for CI with the Brief Neuropsychological Cognitive Examination (BNCE). We evaluated some of the cognitive measures using the TMT test. The first part A of the TMT test is used primarily to examine cognitive processing speed. The second part B of the test, in which the subject alternates between numbers and letters, is used to examine executive functioning.

Results: CI according to the BNCE was identified in 28 patients (43.75\%) and corresponded to mild cognitive deficit (Figure 1). Patients with CI were characterized by low scores on the subtests «set of sequences» (patients with CI $2 \pm 0.21$; patients without CI $-2.67 \pm 0.44 ; \mathrm{p}<0.001)$ and «working memory» $(2.1 \pm 0.57 ; 2.89 \pm 0.2 ; \mathrm{p}<0.001)$ which indicate a decrease in working memory. Patients with CI had also attention deficits $(1.89 \pm 0.26 ; 2.67 \pm 0.44 ; \mathrm{p}<0.001)$.

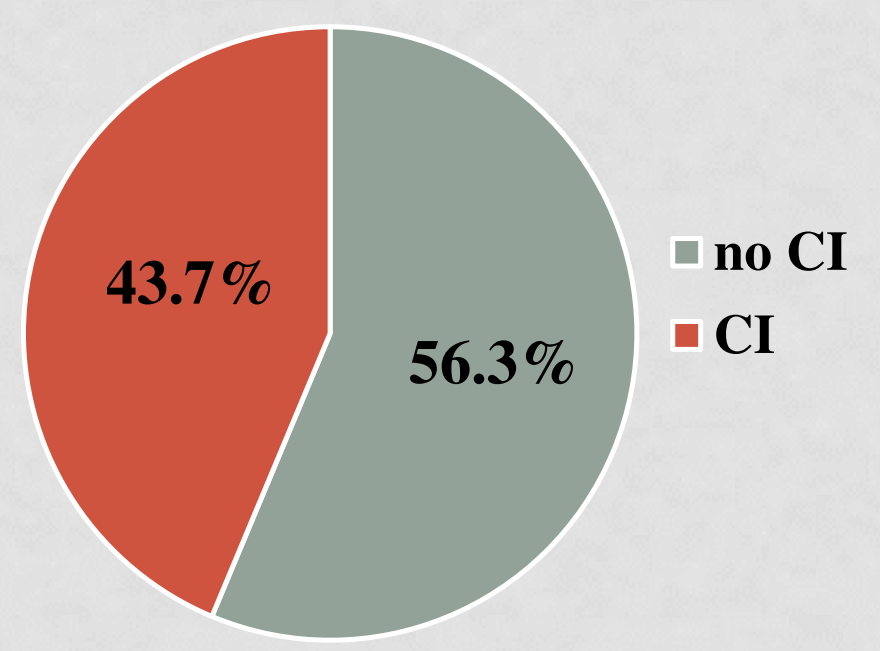

Figure 1. Cognitive impairment in patients with BD in remission.
The TMT test also showed the presence of statistically significant $(\mathrm{p}<0.001)$ attention deficits in patients with CI (Figure 2). CI manifested in a decrease in processing speed, impaired switching and attention distribution.

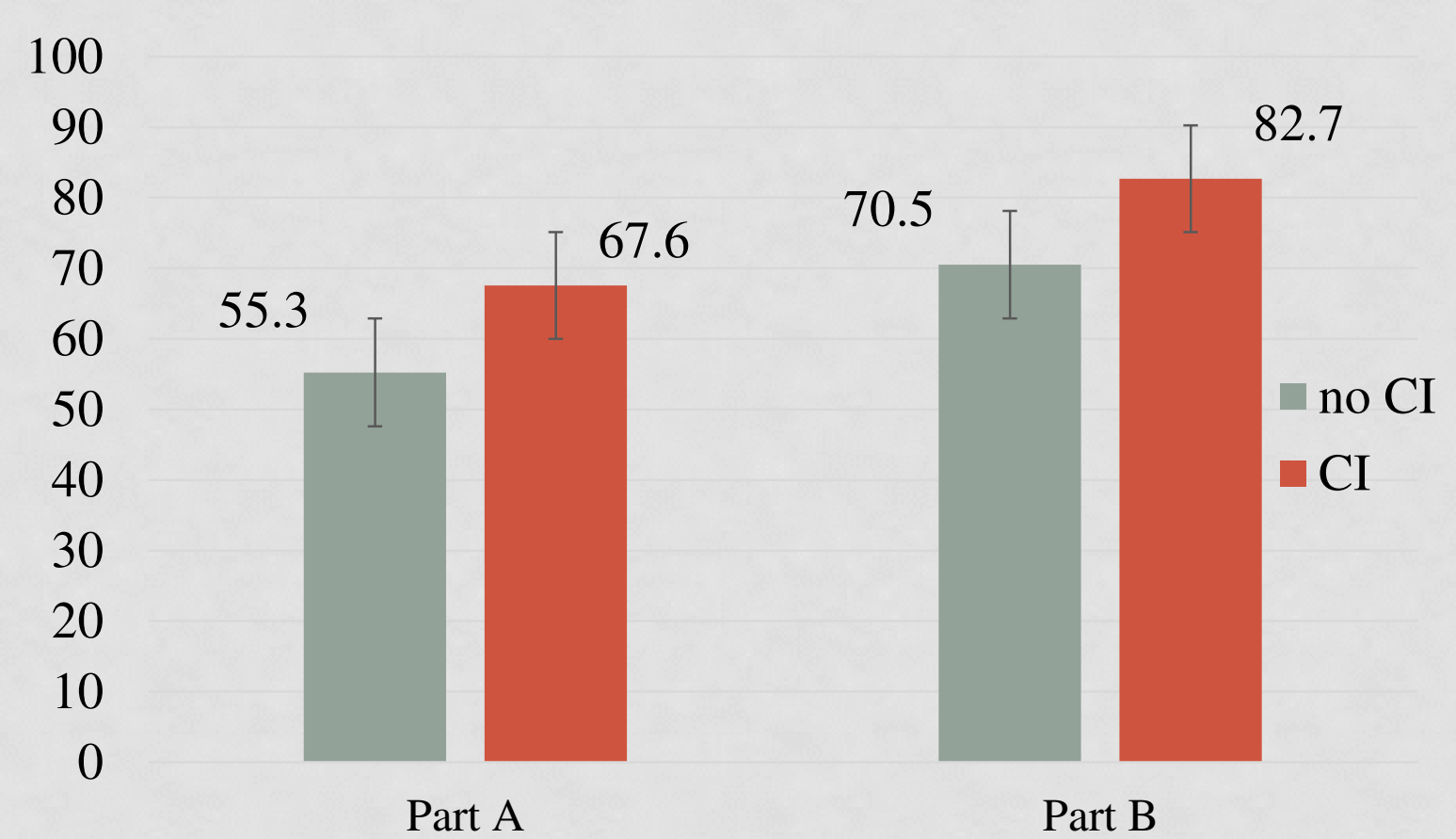

Figure 2. Results of TMT-test

Residual symptoms were observed in $42.2 \%$ of patients in current remission (Table 1). CI was linked with the presence of residual symptoms in remission $(r=0.4 ; p=0.001)$.

\begin{tabular}{|l|c|}
\hline \multicolumn{1}{|c|}{ Residual symptoms } & $\%$ \\
\hline Sleep disturbances & 20.3 \\
\hline Lost of appetite & 4.7 \\
\hline Mental exhaustion & 7.8 \\
\hline $\begin{array}{l}\text { Transitory affective fluctuations (individual depressive } \\
\text { symptoms, subsyndromal depression, etc.) }\end{array}$ & 7.8 \\
\hline $\begin{array}{l}\text { Psychological experiences associated with the } \\
\text { expectation of an acute phase }\end{array}$ & 4.7 \\
\hline
\end{tabular}

Table 1.Residual symptoms in patients with $\mathrm{BD}$ in remission

Conclusions: $\mathrm{CI}$ and residual symptoms occur in over $40 \%$ of patients with BD in remission. The detecting of both $\mathrm{CI}$ and residual symptoms in patients was statistically significant linked in our study. CI in BD should be considered as a potential therapeutic target. This data indicate the need to develop new approaches to BD treatment in remission. 